\title{
Protein and DNA nanoparticulate multiantigenic vaccines against $H$. pylori: in vivo evaluation
}

\author{
Lara Figueiredo $^{1,2}$, Cecília C.R. Calado ${ }^{2}$, António J. Almeida ${ }^{1}$, Lídia M.D. Gonçalves ${ }^{1}$ \\ ${ }^{I}$ Faculdade de Farmácia, Universidade de Lisboa iMed.UL \\ Lisboa,Portugal; (lgoncalves@ff.ul.pt / aalmeida@ff.ul.pt) \\ ${ }^{2}$ Faculdade de Engenharia da Universidade Católica Portuguesa, \\ Rio de Mouro,Portugal (c.calado@fe.lisboa.ucp.pt)
}

\begin{abstract}
Immunisation against $H$. pylori is an attractive option for antibiotic resistance and reinfection situations. Strain genetic heterogeneity, and low immunogenicity of protein antigens and DNA alone are nonetheless obstacles to this approach. We developed multigenic $H$. pylori DNA-nanoparticle and proteinnanoparticle vaccines based on pathogenic relevance. Six antigens were chosen for the vaccine construction: $\operatorname{CagA}$, VacA, HpaA, UreB, HomB and GroEL. Different combinations of CS/DS and CS/AIg /TPP nanoparticles with DNA and chimeric proteins were produced as vaccine systems. Immune responses were evaluated after i.m. and oral immunisation of $B A L B / c$ mice. Oral vaccination successfully stimulated mucosal immunity while i.m. immunisation efficiently elicited a more equilibrated cellular/humoral immune response.
\end{abstract}

Index Terms—nanoparticles; chitosan; vaccine; Helicobacter pylori.

\section{CONTEXT}

Helicobacter pylori infection affects at least half the world's population and implies a high risk of peptic ulcer disease, gastric adenocarcinoma, and mucosa-associated lymphoid tissue lymphoma (Bégué \& Sadowska-Krowicka, 2010; Wilson \& Crabtree, 2007). Antibiotics treatment is successful in most cases but increasing resistance, reinfection, patient compliance and high associated costs ask for preventive measures such as vaccination (Liu et al., 2011).

However the development of an efficient vaccine is impaired by the strain genetic microheterogeneity, each patient being colonized by a cloud of closely related organisms (Vitoriano et al., 2011). In order to overcome the strain genetic heterogeneity, a multiantigenic $H$. pylori vaccine was developed based on fragments of the following six antigens: CagA, VacA, HpaA, UreB, HomB and GroEL. The mouse immunisation strategy, with this multi-epitope vaccine, followed a prime-boost regime based on a DNA-vaccine and protein-vaccine, respectively. DNA vaccines provide several important advantages over current vaccine strategies such as live or attenuated vaccines, as they mimic the effects of natural infection in their ability to endogenously express foreign proteins, also having the ability to induce humoral as well as cellular immune responses (Zhou et al., 2009). A particlemediated delivery system, conceived to protect the antigens degradation while acting as adjuvant, was based on chitosan nanoparticles since this polymer is biocompatible and biodegradable (Cadete et al., 2011).
The oral and the intramuscular (i.m.) routes of immunisation were tested, since bacteria primarily colonize the gastric mucosa, the production of antibodies in the mucosa would constitute an important primary line of defense. Based on the analysis of the Th2 interleucines IL- 4 and IL-10, the Th1 interleucines IFN- $\gamma$ and IL-2, and IgA in the gut, it was observed that the oral immunisation presents promising results pointed out by the mucosa specific IgA levels. However this type of immunisation leads to a less efficient production of specific serum antibodies when compared with i.m. delivery. Hopefully, the present multi-functional vaccine associated to the immunisation protocol developed will strongly contribute to a protective immunogenic response.

\section{GOALS}

\section{A. Protein and DNA chitosan nanoparticle characterization}

Preparing nanoparticles by ionotropic gelation and performing physico-chemical characterization.

\section{B. In vivo sudies}

Immunising $\mathrm{BALB} / \mathrm{c}$ mice with different nanoparticle formulations. Assessing immune response through $\mathrm{IgG}$, IgG1 and IgG2 levels. Evaluating mucosal immune response and quantification of cytokine levels after splenocyte stimulation with antigen.

\section{MATERIALS AND METHODS}

\section{A. Materials}

Chitosan (CS) low molecular weight (LMW) with degree of deacetylation $75-85 \%$, sodium alginate, sodium deoxycholate (DS) and sodium tripolyphosphate (TPP) were obtained from Sigma Aldrich (Dorset, UK).

\section{B. Animals}

Female BALB/c mice ( $\mathrm{n}=5$ /group), 6-8weeks old provided with food and drink ad libitum, were used in the in vivo studies, which were performed in strict accordance with Directive of 24 November ( ${ }^{\circ} 86 / 609$ EEC), the Portuguese laws D.R. $n^{\circ}$ 31/92, D.R. 153 I-A 67/92, and all following legislations. 


\section{Plasmid production and purification}

E. coli XL-1 Blue strain cells were transformed with 1023982 pVax1-Gene4 pVAX1 plasmid according to Cadete et al. (2011). Plasmid purification was performed according the procedure of Maxi Quialfilter Kit (Quiagen, Germany).

\section{Protein Production}

E. coli XL-1 Blue strain, harbouring the recombinant pQE30/CUHpVGHo were used to produce recombinant protein as previously described by Caldeira et al.(2009).

\section{E. Nanoparticle preparation}

The CS/DS (1:1/w:w) and CS/Alg/TPP (LMW/LV) (20:1:0.25/w:w:w) nanoparticles were prepared using an ionotropic gelation technique.

For the (CS/DS) chitosan and sodium deoxycholate were separately dissolved in ultra-pure water in order to obtain $1 \mathrm{mg} / \mathrm{ml}$ solutions. Nanoparticles were formed when 1 volume of DS solution was added drop wise to one volume of CS solution and magnetically stirred at $300 \mathrm{rpm}$ during $15 \mathrm{~min}$ at room temperature as described by Cadete et al. (2011). CS/Alg/TPP nanoparticles formation was carried out according to Gonçalves et al. (2011), whereby TPP containing sodium alginate was added to LMW chitosan in water under magnetic stirring. For the nanoencapsulation, pDNA or protein was added to DS solution or to Alg/TPP solution before the addition to CS solution.

\section{F. Nanoparticle characterization}

Size and $\zeta$-potential measurements were performed by photon correlation spectroscopy and laser Doppler anemometry, respectively, with a Zetasizer Nano-S and Zetasizer 2000 (Malvern Instruments, UK), respectively. All measurements were performed in triplicate. Results were expressed as mean \pm standard deviation (S.D.).

\section{G. Immunisation studies}

Four groups of female BALB/c were immunized on day 1 and boosted on day 22 , with $50 \mu \mathrm{g}$ of protein or DNA via oral or i.m. administration. Controls consisted of non-encapsulated protein and DNA. Blood samples were collected every 15 days, from the tail vein.

Serum specific IgG, IgG1, IgG2a antibodies were detected by ELISA as previously described by Florindo et al. [4] with slight alterations. In order to examine the mucosal immune response, intestines were collected from ethically sacrificed animals and IgA presence assessed by ELISA. Titres are reported as the reciprocal of serum dilutions that gave an optical density $5 \%$ higher than the strongest negative control reading.

\section{RESUlTS AND DISCUSSION}

\section{A. Nanoparticle characterization}

Four nanoparticulate formulations were produced, CS/DS nanoparticles were used for i.m. vaccination and CS/Alg/TPP employed as oral immunisation agent. These systems characteristics are summarized in Table 1:
TABLE I.

NANOPARTICLE VACCINE CHARACTERIZATION. VALUES ARE REPRESENTED AS MEAN \pm S.D.

\begin{tabular}{|c|c|c|c|c|}
\hline \multicolumn{2}{|c|}{ Nanoparticle } & Mean size (nm) & $\begin{array}{c}\text { pDNA/CS } \\
(\boldsymbol{W} / \boldsymbol{W})\end{array}$ & $\begin{array}{c}\text { Encapsulation } \\
\text { efficiency (\%) }\end{array}$ \\
\hline \multirow{2}{*}{ CS/DS } & pDNA & $242 \pm 8$ & 32 & 100 \\
\cline { 2 - 5 } & Protein & $894 \pm 6$ & - & $70 \pm 10$ \\
\hline \multirow{2}{*}{$\begin{array}{c}\text { CS/Alg } \\
\text { /TPP }\end{array}$} & pDNA & $195 \pm 1$ & 15 & 100 \\
\cline { 2 - 5 } & Protein & $328 \pm 7$ & - & $70 \pm 8$ \\
\hline
\end{tabular}

\section{B. Immunisation studies}

Immunogenicity of nanoparticles containing protein and pDNA is confirmed by the increasing systemic levels of specific IgG, IgG1 and IgG2a after i.m. immunisation (Fig. 1). A preliminary assessment of systemic titers of specific antibodies reveals a better immune response towards the i.m. immunization. Moreover, protein nanoparticles present better results than pDNA nanoparticles.
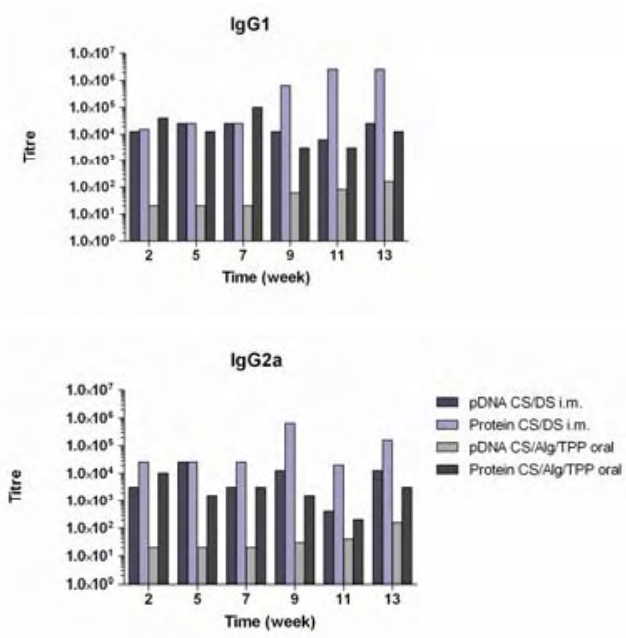

Figure 1 - Serum specific IgG1 and IgG2a titres induced after mice immunisation by i.m. and oral route with nanoparticles encapsulating $S$. equi antigens. Data represent a preliminary assessment concerning serum pool of each group.

Cytokine levels support evidence for a more relevant immune response towards the i.m. immunisation. I.m. immunisation with the encapsulated protein results in a wellequilibrated immune response represented by the presence the characteristic Th1/Th2 cytokines.

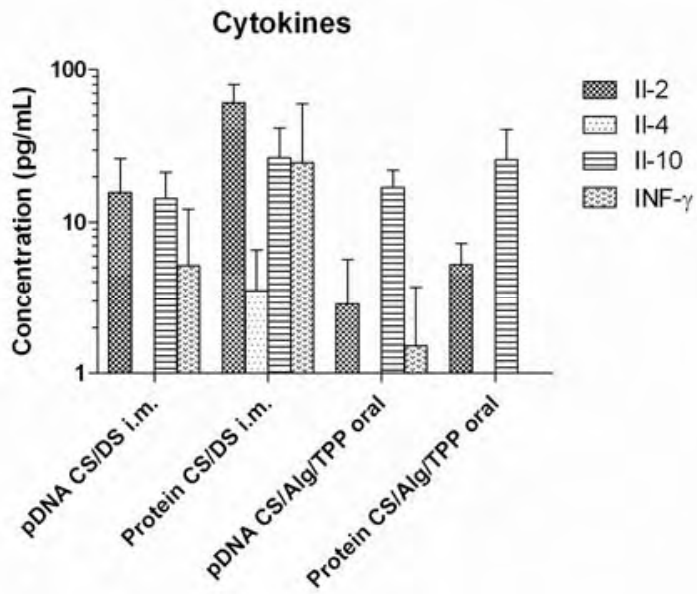

Figure 2 - Cytokine levels after splenocytes stimulation with antigen. 
SIgA levels were also evaluated and revealed an efficient stimulation of mucosal immunity after oral vaccination, presenting statistically different results from the i.m. immunisation.

\section{CONCLUSIONS}

In conclusion, the present study confirms the potential of CS nanoparticles as an adjuvant for DNA vaccines and chimeric protein antigens. Properties of nanoparticles allow a well-equilibrated immune response, showing their potential as a suitable alternative formulation for antigen delivery. CSbased nanoparticle formulations also facilitate mucosal immunity after oral immunisation.

\section{PlanNED DEVELOPMENTS}

In terms of long-term assessment we intend to perform a challenge study, in order to determine the efficacy of the new vaccine formulations.

\section{ACKNOWLEDGMENT}

Work supported by PTDC/BIO/69242/2006 and PEstOE/SAU/UI4013/2011 from FCT and FEDER.

\section{REFERENCES}

[1] R.E. Bégué, H. Sadowska-Krowicka, "Protective efficacy of recombinant urease $\mathrm{B}$ and aluminum hydroxide against Helicobacter pylori infection in a mouse model", FEMS Immunol Med Microbiol, 2010 Nov; 60(2):142-6.

[2] A. Cadete, L. Figueiredo, R. Lopes, C.C. Calado, A.J. Almeida, L.M. Gonçalves, "Development and characterization of a new plasmid delivery system based on chitosan-sodium deoxycholate nanoparticles", Eur J Pharm Sci, 2011 Oct 1, in press.

[3] R.L. Caldeira, L.M. Gonçalves, T.M. Martins, H. Silveira, C. Novo, V. Rosário, A. Domingos, "Plasmodium chabaudi: expression of active recombinant chabaupain-1 and localization studies in Anopheles sp.", Exp Parasitol, 2009 Jun;122(2):97-105.

[4] H.F. Florindo, S. Pandit, L.M. Gonçalves, H.O. Alpar, A.J. Almeida, "Streptococcus equi antigens adsorbed onto surface modified polyepsilon-caprolactone microspheres induce humoral and cellular specific immune responses" Vaccine, 2008 Aug 5;26(33):4168-77.

[5] L.M.D. Goncalves, A. Cadete, L. Figueiredo, C.C.R. Calado, A.J. Almeida, "Biodegradable nanoparticles of alginate and chitosan as nonviral DNA oral delivery system" 1st Portuguese Meeting in Bioengineering (ENBENG), 1-4 March 2011; 1-4.

[6] K.Y. Liu, Y. Shi, P. Luo, S. Yu, L. Chen, Z. Zhao, X.H. Mao, G. Guo, C. $\mathrm{Wu}$, Q.M. Zou, "Therapeutic efficacy of oral immunization with attenuated Salmonella typhimurium expressing Helicobacter pylori CagA, VacA and UreB fusion proteins in mice model". Vaccine, 2011 Sep 2;29(38):6679-85.

[7] I. Vitoriano, A. Rocha-Gonçalves, T. Carvalho, M. Oleastro, C.R Calado, M. Roxo-Rosa, "Antigenic diversity among Portuguese clinical isolates of Helicobacter pylori" Helicobacter, 2011 Apr;16(2):153-68.

[8] K.T. Wilson, J.E. Crabtree, "Immunology of Helicobacter pylori: insights into the failure of the immune response and perspectives on vaccine studies", Gastroenterology, 2007 Jul;133(1):288-308.

[9] W.Y. Zhou, Y. Shi, C. Wu, W.J. Zhang, X.H. Mao, G. Guo, H.X. Li, Q.M. Zou, "Therapeutic efficacy of a multi-epitope vaccine against Helicobacter pylori infection in BALB/c mice model", Vaccine, 2009 Aug 6;27(36):5013-9. 